\title{
OPINIÃO DE ACADÊMICOS DE ENFERMAGEM SOBRE O USO DE PRESERVATIVOS
}

\author{
André Estevam Jaques ${ }^{1}$ \\ Ingrid Mayara Almeida Valera² \\ William Rodrigues de Araujo Zaramello ${ }^{3}$ \\ Franciele de Souza Dantas ${ }^{4}$ \\ Ederson Camilo Pãoeagua ${ }^{5}$ \\ Adalberto Ramon Valderrama Gerbasi ${ }^{6}$
}

JAQUES, A. E.; VALERA, I. M. A.; ZARAMELLO, W. R. de A.; DANTAS, F. de S.; PÃOEAGUA, E. C.; GERBASI, A. R. V. Opinião de acadêmicos de enfermagem sobre o uso de preservativos. Arq. Ciênc. Saúde UNIPAR, Umuarama, v. 18, n. 2, p. 95-100, maio/ago. 2014.

\begin{abstract}
RESUMO: Este estudo tem como objetivo identificar a opinião dos acadêmicos do curso de graduação em Enfermagem sobre a utilização de preservativos, além de oportunizar a este um momento de reflexão sobre a importância da discussão do tema. Para tanto, realizou-se um estudo descritivo, com abordagem quantitativa. A coleta de dados ocorreu em uma universidade do noroeste do Paraná, no período de abril a maio de 2012, por meio de um questionário constituído de duas partes, a primeira abordava dados sóciodemográficos e a segunda tratava questões referentes ao uso de preservativos. Participaram deste estudo 130 acadêmicos. Os resultados evidenciam que os acadêmicos, tanto homens quanto mulheres, discordaram que os preservativos diminuem o prazer sexual; acreditam que os dispositivos devem ser usados em todas as relações sexuais, mesmo com quem se ama e; discordaram que o uso simultâneo de dois preservativos gere maior segurança. Em face aos resultados obtidos e considerando a sexualidade como parte indissociável do ser humano, e a enfermagem em seu papel sócioeducacional, inclusive para as questões relacionadas à saúde sexual e reprodutiva, conclui-se que a abordagem do tema nas universidades é fundamental para a formação de profissionais comprometidos com a manutenção do bem estar social.
\end{abstract}

PALAVRAS-CHAVE: Preservativos; Sexualidade; Enfermagem.

\section{OPINION OF NURSING UNDERGRADUATES ON THE USE OF CONDOMS}

\begin{abstract}
This study aims to identify the opinion of the undergraduate students in nursing on the use of condoms, and create opportunities for a moment of reflection on the importance of such discussions on the theme. Therefore, a descriptive study was carried out with a quantitative approach. Data collection occurred in a university in northwestern Paraná, from April to May 2012, through a questionnaire consisting of two parts, the first addressed socio-demographic data and the second presented issues related to condom use. The study included 130 students. The results show that the students, both men and women, disagreed that the use of condoms reduce sexual pleasure; believe that the devices should be used in all sexual relations, even with someone you love; and disagreed that the simultaneous use of two condoms generate greater security. In view of the results obtained and considering sexuality as an integral part of human beings, and considering nursing in its socio-educational role, even on issues related to sexual and reproductive health, it can be concluded that the approach to the subject in universities is critical for the training of professionals committed to the maintenance of social well-being.
\end{abstract}

KEYWORDS: Condoms; Sexuality; Nursing.

\section{Introdução}

Segundo a Organização Mundial de Saúde (OMS), anualmente são registrados mais de 340 milhões de novos casos de Doenças Sexualmente Transmissíveis (DST) no mundo, ou seja, aproximadamente um milhão de pessoas adquirem DST diariamente, sendo que os jovens e adolescentes são responsáveis por grande parcela desses casos (WHO, 2007; BRASIL, 2011).

A taxa elevada de DST entre jovens pode estar relacionada com o início da atividade sexual precocemente, com a multiplicidade de parceiros sexuais, com relações sexuais desprotegidas e, com o uso de álcool e drogas ilícitas. $\mathrm{O}$ aumento significativo dessas doenças constitui-se em um grave problema de saúde pública, principalmente em relação à infeção do vírus da AIDS (GUBERT; MADUREIRA, 2009; PEREIRA et al., 2014).
Por essa razão, o desenvolvimento de programas de prevenção para este grupo populacional é considerado um grande desafio, inclusive no que tange a interação com jovens que não têm acesso aos serviços de saúde e que, portanto, podem se encontrar em situações de vulnerabilidade às DST (PAIVA et al., 2008).

Nesse sentido, na vida sexual e reprodutiva, os preservativos, tanto masculinos, quanto femininos, constituem-se em dispositivos de barreira para proteção de homens e mulheres, proporcionando dupla função de proteção contra inúmeras DST e gravidez indesejada. Entretanto, as resistências quanto ao seu uso, sejam explícitas ou mesmo veladas, são comuns (SILVA et al. 2014).

$\mathrm{O}$ aumento do uso de preservativos ocorreu em decorrência da epidemia da AIDS, na década de 1980 e, foi intensificada na década de 1990, contudo, alguns estudos evidenciam que, atualmente, apesar do conhecimento razo-

DOI: https://doi.org/10.25110/arqsaude.v18i2.2014.5173

${ }^{1}$ Enfermeiro. Doutor em Ciências pela Escola de Enfermagem de Ribeirão Preto - EERP/USP. Professor titular da Universidade Paranaense - UNIPAR. E-mail: aejk2002@yahoo.com.br

${ }^{2}$ Enfermeira. Mestranda em Enfermagem pela Universidade Estadual de Maringá - UEM. E-mail: ingrid_imav@hotmail.com

${ }^{3}$ Enfermeiro. Especializando em Enfermagem nas Emergências pela Pontifícia Universidade Católica do Paraná, PUC/PR. E-mail: williamzaramello@ hotmail.com

${ }^{4}$ Enfermeira. Coordenadora no Centro de Reabilitação Integral Dom Aquino Corrêa - CRIDAC. E-mail: fran.0809@hotmail.com

${ }^{5}$ Enfermeiro. Graduando em Filosofia pela Pontifícia Universidade Católica do Paraná, PUC/PR. E-mail: eder.camilo@hotmail.com

${ }^{6}$ Matemático. Doutor em Inovação Educativa pela Universidade Autônoma de Barcelona. Professor da Universidade Paranaense - UNIPAR. E-mail: gerbasi@unipar.br 
ável existente sobre DST, seu índice de utilização ainda é insatisfatório, mesmo diante do quadro epidemiológico vigente (MADUREIRA; TRENTINI, 2008; PASCOM; ARRUDA; SAKITA, 2011).

Nesse cenário, os profissionais de saúde, sobretudo enfermeiros, se destacam como fortes aliados no combate às DST por promoverem o cuidado em vários setores da saúde, sendo, dessa maneira, fundamental que sejam capacitados, já nas universidades, para tal finalidade e, possibilitem a troca de saberes, a educação e a sensibilização da população para os benefícios da adoção de comportamentos seguros, como o uso de métodos preventivos (LUNA et al., 2013).

A prevenção, por meio do uso de preservativos, ainda é a conduta mais eficaz no combate às DST, logo, este estudo se justifica ao considerar o importante envolvimento de enfermeiros em práticas educativas, voltadas à redução dos índices alarmantes de tais infecções, junto à população. Dessa forma, constituiu-se a seguinte questão de pesquisa, que norteou este estudo: Qual a opinião dos acadêmicos de enfermagem sobre a utilização do preservativo?

Nesse contexto, esta pesquisa objetivou identificar a opinião dos acadêmicos do curso de graduação em Enfermagem sobre a utilização de preservativos, além de oportunizar a este um momento de reflexão sobre a importância da discussão da temática em apreço.

\section{Material e Método}

A pesquisa desenvolvida foi de caráter descritivo e de natureza quantitativa. A população alvo foi constituida por 172 acadêmicos do curso de graduação em Enfermagem de uma universidade do noroeste do Paraná (Brasil), sendo que, o tamanho da amostra calculada foi igual a 130 acadêmicos. Assim, utilizou-se o processo de amostragem aleatória simples para escolher os sujeitos que participaram da pesquisa.

A coleta de dados foi realizada no período de 01 de abril a 30 de maio de 2012, em sala de aula. O critério de inclusão adotado para participar deste estudo foi de forma voluntária por parte do acadêmico, sendo que este deveria estar regularmente matriculado no curso.

Para a coleta de dados utilizou-se questionário contituído de duas partes, a primeira abordava os dados sócio- -demográficos do acadêmico (sexo, idade, estado civil, crença religiosa, ano do curso e série que frequenta no curso), enquanto que a segunda, tratava questões referentes ao uso de preservativos masculino e feminino. $\mathrm{O}$ instrumento foi adaptado do modelo CAP 7.0 de pesquisadoras da Universidade Estadual de São Paulo/Instituto de Psicologia/Núcleo de Estudo para Prevenção da AIDS (USP/NEPAIDS). Os resultados foram tabulados com planilha do programa Microsoft Excel ${ }^{\circledR}$ versão 2010 e analisados com amparo da literatura adequada.

Os dados foram coletados após a apreciação do Comitê de Ética em Pesquisa Envolvendo Seres Humanos da universidade, sob protocolo $\mathrm{n}^{\circ} 21453 / 2012$, aprovado em 01/08/2011 e mediante assinatura do Termo de Consentimento Livre e Esclarecido pelos participantes, respeitando os preceitos éticos-legais da experimentação humana.

\section{Resultados}

Participaram da pesquisa 130 acadêmicos do curso de graduação em enfermagem, sendo que desses, 76,92\%, que corresponde a 100 alunos, eram do sexo feminino e, $23,08 \%$, que somam 30 alunos, eram do sexo masculino, dado que chama atenção ao corroborar com a literatura, que aponta que a predominância de mulheres nos cursos de enfermagem se deve à 'constituição histórico-social' que ainda rege fortemente a profissão (CORRÊA et al., 2011).

Quanto à distribuição dos acadêmicos por faixa etária, observou-se que a grande maioria tinha entre 21 e 25 anos (45,38\%); seguido por 16 a 20 anos (38,46\%); 30 a 34 anos $(13,85 \%)$ e; 35 ou mais $(2,31 \%)$. No que tange ao ano que frequentava do curso, $31,54 \%$ eram do $1^{\circ}$ ano; $20 \%$ do $4^{\circ}$ ano; $17,69 \%$ correspondiam ao $3^{\circ}$ e $2^{\circ}$ ano e; $13,08 \%$ ao $5^{\circ}$ ano. No que se refere ao estado civil, $82,30 \%$ eram solteiros; $11,54 \%$ casados; $3,08 \%$ divorciados; $2,31 \%$ não responderam e; apenas um $(0,77 \%)$ se declarou viúvo. Quanto à crença religiosa, $81,53 \%$ se declararam católicos; $11,54 \%$ evangélicos; 3,85\% não responderam; 2,31\% espíritas e; $0,77 \%$ se revelou ateu.

Os dados referentes à opinião dos acadêmicos de enfermagem sobre uso de preservativo encontram-se dispostos na Tabela 1.

Tabela 1: Opinião de acadêmicos de enfermagem sobre o uso de preservativos. Umuarama, Paraná, Brasil, 2014.

\begin{tabular}{|c|c|c|c|c|c|c|c|c|c|c|}
\hline \multirow{3}{*}{ Questões } & \multirow{2}{*}{\multicolumn{2}{|c|}{$\begin{array}{c}\begin{array}{c}\text { Discordo } \\
\text { Totalmente }\end{array} \\
\text { n (\%) }\end{array}$}} & \multicolumn{2}{|c|}{ Discordo } & \multicolumn{2}{|c|}{$\begin{array}{l}\text { Nem concordo } \\
\text { nem discordo }\end{array}$} & \multicolumn{2}{|c|}{ Concordo } & \multicolumn{2}{|c|}{$\begin{array}{l}\text { Concordo } \\
\text { Totalmente }\end{array}$} \\
\hline & & & & & & $\%)$ & & & n ( & \\
\hline & $\mathbf{F}$ & M & $\mathbf{F}$ & $\mathbf{M}$ & $\mathbf{F}$ & M & $\mathbf{F}$ & M & $\mathbf{F}$ & M \\
\hline $\begin{array}{l}\text { Os preservativos } \\
\text { diminuem o prazer } \\
\text { sexual? }\end{array}$ & $\begin{array}{c}19 \\
(14,62)\end{array}$ & $02(1,54)$ & $\begin{array}{c}34 \\
(26,15)\end{array}$ & $10(7,69)$ & $\begin{array}{c}25 \\
(19,23)\end{array}$ & $08(6,15)$ & $\begin{array}{c}17 \\
(13,08)\end{array}$ & $07(5,38)$ & $05(3,85)$ & $03(2,31)$ \\
\hline $\begin{array}{l}\text { O uso de } \\
\text { preservativos } \\
\text { atrapalham as } \\
\text { relações sexuais? }\end{array}$ & $\begin{array}{c}20 \\
(15,38)\end{array}$ & $07(5,38)$ & $\begin{array}{c}49 \\
(37,70)\end{array}$ & $\begin{array}{c}14 \\
(10,77)\end{array}$ & $\begin{array}{c}21 \\
(16,15)\end{array}$ & $03(2,31)$ & $08(6,15)$ & $04(3,08)$ & $02(1,54)$ & $02(1,54)$ \\
\hline $\begin{array}{l}\text { Os preservativos não } \\
\text { são dispendiosos? }\end{array}$ & $12(9,23)$ & $07(5,38)$ & $\begin{array}{c}25 \\
(19,23)\end{array}$ & $10(7,69)$ & $\begin{array}{c}33 \\
(25,39)\end{array}$ & $08(6,15)$ & $\begin{array}{c}18 \\
(13,85)\end{array}$ & $02(1,54)$ & $12(9,23)$ & $03(2,31)$ \\
\hline $\begin{array}{l}\text { Os preservativos são } \\
\text { nojentos? }\end{array}$ & $\begin{array}{c}26 \\
(20,00)\end{array}$ & $\begin{array}{c}16 \\
(12,31)\end{array}$ & $\begin{array}{c}53 \\
(40,77)\end{array}$ & $08(6,15)$ & $10(7,69)$ & $04(3,08)$ & $09(6,92)$ & $02(1,54)$ & $02(1,54)$ & - \\
\hline
\end{tabular}




\begin{tabular}{|c|c|c|c|c|c|c|c|c|c|c|}
\hline $\begin{array}{l}\text { Os preservativos } \\
\text { são fáceis de serem } \\
\text { colocados? }\end{array}$ & - & $01(0,77)$ & $09(6,92)$ & $02(1,54)$ & $\begin{array}{c}16 \\
(12,31)\end{array}$ & $05(3,85)$ & $\begin{array}{c}60 \\
(46,15)\end{array}$ & $\begin{array}{c}14 \\
(10,77)\end{array}$ & $\begin{array}{c}15 \\
(11,54)\end{array}$ & $08(6,15)$ \\
\hline $\begin{array}{l}\text { Os preservativos } \\
\text { podem ser } \\
\text { usados sem } \\
\text { constrangimentos? }\end{array}$ & - & $01(0,77)$ & $06(4,61)$ & $03(2,31)$ & $07(5,38)$ & $03(2,31)$ & $\begin{array}{c}55 \\
(42,31)\end{array}$ & $11(8,46)$ & $\begin{array}{c}32 \\
(24,62)\end{array}$ & $12(9,23)$ \\
\hline $\begin{array}{l}\text { Eu tenho vergonha } \\
\text { de comprar } \\
\text { preservativos. }\end{array}$ & $\begin{array}{c}27 \\
(20,77)\end{array}$ & $\begin{array}{c}18 \\
(13,85)\end{array}$ & $\begin{array}{c}32 \\
(24,62)\end{array}$ & $07(5,38)$ & $11(8,46)$ & $03(2,31)$ & $\begin{array}{c}21 \\
(16,15)\end{array}$ & $02(1,54)$ & $09(6,92)$ & - \\
\hline $\begin{array}{l}\text { Preservativos não } \\
\text { precisam ser usados } \\
\text { com quem se ama? }\end{array}$ & $\begin{array}{c}43 \\
(33,07)\end{array}$ & $\begin{array}{c}21 \\
(16,15)\end{array}$ & $\begin{array}{c}41 \\
(31,54)\end{array}$ & $05(3,85)$ & $10(7,69)$ & $02(1,54)$ & $04(3,08)$ & $01(0,77)$ & $02(1,54)$ & $01(0,77)$ \\
\hline $\begin{array}{l}\text { É mais seguro usar } \\
\text { dois preservativos } \\
\text { masculinos durante } \\
\text { as relações sexuais? }\end{array}$ & $\begin{array}{c}42 \\
(32,31)\end{array}$ & $\begin{array}{c}21 \\
(16,15)\end{array}$ & $\begin{array}{c}33 \\
(25,39)\end{array}$ & $07(5,38)$ & $\begin{array}{c}16 \\
(12,31)\end{array}$ & $02(1,54)$ & $08(6,15)$ & - & $01(0,77)$ & - \\
\hline $\begin{array}{l}\text { Por medida de } \\
\text { segurança contra } \\
\text { as DST, deve-se } \\
\text { usar o preservativo } \\
\text { masculino e o } \\
\text { feminino ao mesmo } \\
\text { tempo? }\end{array}$ & $\begin{array}{c}35 \\
(26,93)\end{array}$ & $\begin{array}{c}15 \\
(11,54)\end{array}$ & $\begin{array}{c}44 \\
(33,85)\end{array}$ & $08(6,15)$ & $10(7,69)$ & $06(4,61)$ & $06(4,61)$ & $01(0,77)$ & $05(3,85)$ & - \\
\hline
\end{tabular}

\section{Discussão}

Nas últimas décadas, mesmo havendo mudanças sociais e culturais significativas no âmbito da sexualidade, sua vivência na adolescência e juventude continua sendo tratada como uma atividade de risco, associada, quase sempre às DSTs e gravidez precoce (BRANDÃO, 2009). Atendendo a essa problemática, o preservativo é o recurso disponível, à homens e mulheres, que atende à dupla função de prevenção de DST e da gravidez indesejada, contudo, mesmo assim, são comuns as resistências quanto ao seu uso por parte de ambos os sexos (MADUREIRA; TRENTINI, 2008).

$\mathrm{O}$ acesso às políticas de prevenção e orientação sobre a saúde sexual e reprodutiva, bem como o aumento do uso de preservativos, têm sido grandes aliados na redução dos agravos oriundos das relações sexuais desprotegidas. Grande parte dos jovens possui algum conhecimento sobre medidas preventivas, entretanto, aparentemente questões relacionadas à cultura e educação dificultam o emprego de tais ações, como o sentimento de nojo relacionado aos preservativos, ou até mesmo a sensação de redução do prazer e desconforto (VELHO, 2011).

Enfatiza-se ainda, que a vulnerabilidade dos jovens, principalmente os integrantes de cursos superiores ligados à área de saúde, como a enfermagem, se relaciona intimamente com o sentimento de conhecimento sobre o assunto e, esse excesso de autoconfiança pode favorecer a adoção de práticas inseguras e de um comportamento sexual pouco saudável, sendo fundamental o envolvimento da universidade nestas questões (BEZERRA et al., 2012).

No presente estudo, apesar de a maioria dos estudantes discordarem que o uso do preservativo diminui o prazer durante a relação sexual, ainda há um número razoável de estudantes que concordaram com este pensamento, independentemente do sexo. A relação da redução do prazer com o uso de preservativos também é observada em pesquisa que aponta que $31,2 \%$ dos acadêmicos e $30,8 \%$ das acadêmicas de enfermagem concordam que o uso de preservativo diminui o prazer (COSTA et al., 2007).

A crença de que preservativos dificultam o prazer nos permite concluir que a exposição ao risco, de forma consciente, pode ocorrer em função da busca de prazer sexual e que, portanto, muitas vezes o prazer é mais valorizado em detrimento à própria saúde do indivíduo (BARBOSA; FREITAS, 2014). Assim, o conceito de preservativo atrelado à diminuição do prazer e a consequente não satisfação sexual, favorece as relações sexuais desprotegidas e pode interferir nas orientações prestadas pelos acadêmicos e futuros profissionais de enfermagem.

O desenvolvimento da sexualidade, presente em toda trajetória de vida do ser humano, nem sempre é acompanhado de um amadurecimento afetivo e cognitivo, sendo a juventude a fase que reflete a vulnerabilidade a riscos como: gravidez indesejada e DST. Contudo, atualmente reconhece-se que, jovens que usam o preservativo na primeira relação sexual, desenvolvem maior consciência da necessidade do uso e tendem a usá-lo ao longo de sua vida, realizando-o com segurança (ALVES; LOPES, 2008).

Nesse contexto, identificou-se que quase $70 \%$ dos participantes, independentemente do sexo, discordaram que os preservativos atrapalham a relação sexual. Em estudo semelhante, pesquisadores descrevem que $41,6 \%$ dos entrevistados concordam que o uso de preservativo atrapalha o ato sexual, indicando que a ausência do uso desses métodos preventivos, relaciona-se com a dificuldade em se acostumar com o preservativo (COSTA et al., 2007). A literatura descreve que tais comportamentos e relatos devem-se ao fato de o preservativo ainda ser percebido como um objeto que interfere no relacionamento, indicando que a interrupção da atividade sexual para sua colocação pode significar redução do prazer e da espontaneidade da relação (VIEIRA, 2014).

O uso do preservativo envolve uma série de valo- 
res impostos socialmente, tornando importante enfatizar a associação entre o preservativo, o prazer e a segurança, para que prevaleça a negociação e o respeito nas relações sexuais (GOMES et al., 2011).

Observou-se que a maioria dos acadêmicos afirmaram que o preservativo deve ser usado, mesmo com quem se ama. Contudo, ainda percebemos um percentual reduzido, embora preocupante de estudantes, principalmente do sexo feminino $(4,62 \%)$, que concordaram que quando há amor na relação, o preservativo é dispensável, o que nos remete às velhas (ou atuais) relações desiguais de poder entre homens e mulheres, que dificultam a negociação quanto ao uso do preservativo. A confiança no parceiro e a imposição de determinadas normas sociais, interferem negativamente no uso do preservativo (CHAVES et al., 2014).

É importante destacar, que a eficácia deste método preventivo pode estar comprometida devido a defeitos de fabricação que podem ocorrer, embora a causa mais comum de redução da qualidade de proteção do preservativo relaciona-se intimamente com atitudes do próprio usuário, como o local escolhido para armazenamento, sua utilização propriamente dita, de maneira inadequada, mais comumente identificável durante a colocação do preservativo (COSTA et al., 2007).

Notou-se que a maioria dos acadêmicos, principalmente do sexo feminino, considera fácil a colocação do preservativo. $\mathrm{O}$ que vem em contrapartida ao exposto por pesquisadores que referiram como barreira ao seu uso, em especial o feminino, a dificuldade apresentada por algumas mulheres na colocação do dispositivo, ademais, se considera como fator contribuinte ao descarte do preservativo a relutância, principalmente entre mulheres, em aderir a práticas sexuais saudáveis e seguras (ALBUQUERQUE; VILLELA, 2011), o que pode ser explicado pelo fato dos jovens iniciarem sua via sexual cada vez mais cedo e sem fazer uso da camisinha, por não gostar ou por considerá-la constrangedora, e terem vergonha de comprar ou solicitar nos postos de saúde e, mesmo de pedir para o parceiro usar (VIEIRA et al., 2004)

Quanto à opinião dos acadêmicos sobre os preservativos não serem dispendiosos, 3,85\% dos acadêmicos e $23,08 \%$ das acadêmicas concordaram; enquanto que $13,07 \%$ dos acadêmicos e $28,46 \%$ das acadêmicas discordaram. Pesquisa realizada com jovens do gênero feminino, sexualmente ativas, entre 15 e 19 anos revelou que dentre as razões que impedem uso do preservativo, apenas $0,7 \%$ e $1,3 \%$, respectivamente, revelaram que não utilizavam por conta do alto custo e por não saber ou não ter onde comprar (VIEIRA et al., 2004).

Dentre os fatores que podem contribuir para a resistência ao uso do preservativo, podemos citar a crença de diminuição da sensibilidade durante o ato sexual, dificuldade de uso, preço elevado, vergonha e até mesmo desinteresse, que ocorre mais entre o sexo feminino. Outro ponto a ser considerado tem ligação com a falta de acesso, que é agravada pela deficiência de conhecimento da vulnerabilidade, o que torna os preservativos dispendiosos (NASCIMENTO; BARBOSA; MEDRADO, 2005).

É importante considerar, neste sentido, que somente em 2011 foram gratuitamente distribuídos 493 milhões de preservativos masculinos no Brasil e em 2012 cerca de 20 milhões de preservativos femininos foram disponibilizados à população sem qualquer custo (COELHO, 2012).

A saúde sexual e reprodutiva, atualmente, ocupa espaço expressivo dentro das políticas públicas de saúde e educação, devido à sua importância profilática sobre agravos que afetam a saúde e o bem-estar da população de modo geral. Logo, o preservativo surgiu como meio preventivo de baixo custo contra as DST (ROCHA; DIAS; GAMA, 2010).

Sobre o uso de dois preservativos masculinos proporcionar maior segurança à relação sexual, a grande maioria, tanto de acadêmicas, quanto de acadêmicos discordaram. Com a disseminação alarmante dos casos de DST investiu-se na adoção de medidas preventivas seguras e acessíveis, como os preservativos. Todavia, o uso incorreto deste método, que ocorre por falta de informação e treinamento adequados, pode levar ao rompimento do dispositivo e gerar prejuízos ao usuário, colocando-o a mercê de complicações (NETO et al., 2009).

Este cenário representa um grande impacto na saúde reprodutiva e sexual de jovens e adolescentes, predispondo-os a diversos agravos como câncer uterino, infecções e doenças inflamatórias pélvicas, gravidez ectópica, recém-nascidos de baixo peso, esterilidade, o que por sua vez, são fatores de risco para contaminação pelo vírus HIV (WHO, 2007).

Evidenciou-se com esta investigação que algumas acadêmicas acreditam que o uso de dois preservativos masculinos é mais seguro. Em pesquisa similar realizada em Pernambuco aponta que $8,9 \%$ dos estudantes detêm a mesma opinião. Além disso, identificou-se também que muitos acadêmicos, de ambos os sexos, acreditam que o uso simultâneo de preservativos masculino e feminino durante a relação sexual propicia maior segurança, dados que geram preocupação, tanto em relação à saúde dos acadêmicos, quanto à divulgação de informações incorretas por parte destes, e que indicam a necessidade de investimentos em ações educativas direcionadas à sexualidade humana (COSTA et al., 2007).

É responsabilidade dos profissionais de saúde, o esclarecimento à população acerca da utilização correta dos métodos preventivos, uma vez que estes possuem, como uma de suas mais difíceis atribuições, conscientizar a sociedade sobre as questões relacionadas à saúde, que é obtida apenas através da quebra de tabus (NETO et al., 2009).

Nesse contexto, um estudo revela que apenas 55,4\% dos universitários pesquisados, incluindo os da área da saúde, haviam recebido orientação sobre uso de preservativos durante a graduação, o que segundo os autores, deixa em evidência a necessidade de medidas educativas voltadas para estes jovens, não apenas, para que possam adotar práticas seguras, mas para que sejam fontes de informações corretas à população (COSTA; ROSA; BATTISTI, 2009).

A enfermagem, como prática social e assistencial, desenvolve um papel fundamental na promoção e prevenção de agravos relacionados à saúde sexual e reprodutiva, como as DST, principalmente na população jovem (FERREIRA et al., 2012). Deve-se considerar o acadêmico de enfermagem como um futuro profissional e educador em saúde, que irá prestar assistência a pacientes portadores de HIV/AIDS e outras DST, além disso, com sua inserção no contexto do Sistema Único de Saúde (SUS), este será responsável pelo desenvolvimento de ações de promoção, prevenção e assistência a saúde tanto no aspecto coletivo quanto individual 
(COSTA et al., 2007).

De maneira geral, o saber voltado para a sexualidade e seus desdobramentos favorece o autoconhecimento pessoal, social e profissional. Assim, o acadêmico de enfermagem pode desenvolver competências didáticas para um efetivo processo educativo para as questões inerentes à sexualidade, bem como articular propostas e disseminar saberes entre os demais profissionais de saúde e educação que constituem a equipe multiprofissional de saúde (PHILBERT et al., 2011).

\section{Conclusão}

Constatou-se com essa investigação que a maioria dos acadêmicos de enfermagem considera que o uso de preservativos não diminui o prazer sexual. Todavia, pode-se supor que entre os acadêmicos que afirmaram que o dispositvo atrapalha o ato sexual, podem apresentar dificuldade em seu manuseio e utilização, já que quando indagados se os preservativos são fáceis de serem colocados, cerca de $10 \%$ apenas, concordaram com a assertiva.

Quanto à dispendiosidade dos preservativos, a diferença entre o percentual de acadêmicos que concordaram e discordaram foi mínima, embora os que afirmam que os preservativos são dispendiosos assumiram pequena vantagem numérica. Mas, a grande maioria dos estudantes acredita que o aparato precisa ser utilizado em todas as relações sexuais, inclusive com quem se ama.

Nesse sentido, faz-se indispensável afirmar que a eficácia do método depende da observação de alguns fatores, entre eles o seu correto armazenamento, colocação e uso. Evidenciou-se, que os sujeitos reconhecem que o uso de dois preservativos simultaneamente, sejam dois masculinos ou um masculino e um feminino, constituí-se uma prática que compromete as propriedades de resistência do dispositivo, propiciando possível fracasso ao método.

Diante do que fora exposto, e considerando a sexualidade como parte indissociável do ser humano e, levando-se em conta ainda, que os acadêmicos já podem ser fontes de informações à comunidade, inclusive para as questões ligadas à saúde sexual e reprodutiva, concluiu-se que a importância da abordagem do tema em questão pela universidade é fundamental para a formação de profissionais de saúde competentes e comprometidos com a manutenção de estilos de vida saudáveis.

\section{Referências}

ALBUQUERQUE, G.A.; VILLELA, W.V. Uso do preservativo feminino como método contraceptivo: experiências de mulheres em uma Unidade Básica de Saúde no município de Juazeiro do Norte - CE. Rev. APS, v. 14, n. 2, p. 185-96, 2011.

ALVES, A.S.; LOPES, M.H.B.M. Conhecimento, atitude e prática do uso de pílula e preservativo entre adolescentes universitários. Rev bras enferm., v. 61, n. 1, p. 11-17, 2008

BARBOSA, J.A.G.; FREITAS, M.I.F. Percepções de homens com transtornos mentais sobre risco e autocuidado face às Infecções Sexualmente Transmissíveis. Saúde Soc., v. 23, n. 2 , p. 523-35, 2014.

BEZERRA, E.O. et al. Análise da vulnerabilidade sexual de estudantes universitários ao HIV/AIDS. Rev Rene, v. 13, n. 5, p. 1121-31, 2012.

BRANDÃO, E.R. Desafios da contracepção juvenil: interseções entre gênero, sexualidade e saúde. Cien Saúde Colet., v. 14, n. 4, p. 1063-1071, 2009.

BRASIL. Ministério da Saúde. Boletim Epidemiológico Aids e DST 2011. Ano 8 (1). Brasília: Departamento de DST/HIV/AIDS e hepatites virais. 2011. 162 p.

CHAVES, A.C.P. et al. Conhecimentos e atitudes de adolescentes de uma escola pública sobre a transmissão sexual do HIV. Rev Bras Enferm, v. 67, n. 1, p. 48-53, 2014.

COELHO, N. Ministério da Saúde adquire 20 milhões de camisinhas femininas. Ministério da Saúde. Brasília, 2012. Disponível em: Disponível em: http://portal.saude.gov.br/portal/aplicacoes/noticias/ default.cfm?pg $=$ dspDetalheNoticia\&id_area $=124 \& C O$ NOTICIA=13789. Acesso em: 03 out. 2010.

CORRÊA, A.K. et al. Perfil de estudantes ingressantes em licenciatura: Escola de Enfermagem de Ribeirão Preto da Universidade de São Paulo. Rev Esc Enferm USP, v. 45, n 4, p. 933-8, 2011.

COSTA, A.V. et al. Opiniões dos estudantes de enfermagem sobre os preservativos masculino e feminino. Rev enferm UFPB on line., v. 1, n. 1, p. 46-53, 2007. Disponível em: http://www.revista.ufpe.br/revistaenfermagem/index.php/ revista/article/view/11-9078-1-. Acesso em: 11 nov. 2013.

COSTA, L.C.; ROSA, M.I.; BATTISTI, I.D.E. Prevalence of condom use and associated

factors in a sample of university students in southern Brazil. Cad Saúde Pública, v. 25, n. 6, p. 1245-1250, 2009.

FERREIRA, A.G.N. et al. Cultura masculina e religiosidade na prevenção das DST/HIV/AIDS em adolescentes. Reme Rev. Min. Enferm., v. 16, n. 4, p. 572-78, 2012.

GOMES, V.L.O. et al. Percepções de casais heterossexuais acerca do uso da camisinha feminina. Esc. Anna Nery Rev Enferm., v. 15, n. 1, p. 22-30, 2011.

GUBERT, D.; MADUREIRA, V.S.F. Iniciação sexual de homens adolescentes. Ciênc. saúde coletiva, v. 14, n. 4, p. 1119-28, 2009.

LUNA, I.T. et al. Conhecimento e prevenção das Doenças Sexualmente Transmissíveis entre adolescentes em situação de rua. Cienc Cuid Saude, v. 12, n. 2, p. 346-55, 2013.

MADUREIRA, V.S.F.; TRENTINI, M. Da utilização do preservativo masculino à prevenção de DST/aids. Cien Saúde Colet., v. 13, n. 6, p. 1807-1816, 2008. 
NASCIMENTO, A.M.G.; BARBOSA, C.S.; MEDRADO, B. Mulheres de Camaragibe: representação social sobre vulnerabilidade feminina em tempos de AIDS. Rev bras saúde matern infant., v. 5, n. 1, p. 77-86, 2005.

NETO, A.B. et al. Revisão sobre a eficácia do preservativo em relação à proteção contra doenças sexualmente transmissíveis e gestação. Diagn Tratamento, v. 14, n. 3, p. 123-5, 2009.

PAIVA, V. et al. Grupo de Estudos em População, Sexualidade e Aids. Idade e uso de preservativo na iniciação sexual de adolescentes brasileiros. Rev Saúde Pública, v. 42, supl. 1, p. 45-53, 2008.

PASCOM, A.R.P.; ARRUDA, M.R.; SAKITA, K.M. Pesquisa de Conhecimentos, Atitudes e Práticas na População Brasileira, 2008. In: BRASIL. Ministério da Saúde. Secretaria de Vigilância Saúde. Pesquisa de conhecimento, atitudes e práticas na população brasileira de 15 a 64 anos; 2008. Brasília: Ministério da Saúde, 2011.

PEREIRA, B. S. et al. Fatores associados à infecção pelo HIV/AIDS entre adolescentes e adultos jovens matriculados em Centro de Testagem e Aconselhamento no Estado da Bahia, Brasil. Ciênc. saúde coletiva, v. 19 , n. 3, p. 747-58, 2014.

PHILBERT, L.A.S. et al. Investigação da formação dos estudantes do curso de licenciatura em enfermagem para temática sexualidade humana. In: II SIMPÓSIO INTERNACIONAL DE EDUCAÇÃO SEXUAL Gênero, Direitos e Diversidade Sexual: Trajetórias Escolares, 04., 2011, Maringá. Anais do II SIMPÓSIO INTERNACIONAL DE EDUCAÇÃO SEXUAL - Gênero, Direitos e Diversidade Sexual: Trajetórias Escolares. Maringá: UEM, 2011.

ROCHA, C.M.F.; DIAS, S.F.; GAMA, A.F. Conhecimentos sobre o uso de contraceptivos e prevenção de DST: a percepção de mulheres imigrantes. Cad Saúde Pública, v. 26, n. 5, p. 1003-12, 2010.

SILVA, L.V.S. et al. O uso de preservativo e a prevenção de doença sexualmente transmissível na terceira idade. Rev.

Rede Cuidad Saúde, v. 8, n. 1, p. 1-11, 2014.

VELHO, M.T.A.C. et al. Uso de condom entre estudantes universitários do sul do Brasil. Saúde (Santa Maria), v. 37, n. 1, p. 43-54, 2011.

VIEIRA, C.P. Lógicas de risco na experiência da sexualidade juvenil. Fórum Sociológico, v. 24, p. 89-94, 2014.

VIEIRA, M.A.S. et al. Fatores associados ao uso do preservativo em adolescentes do gênero feminino no município de Goiânia. DST j bras doenças sex transm., v. 16, n. 3, p. 77-83, 2004.
WHO. World Health Organization, Department of Reproductive Health and Research. Global strategy for the prevention and control of sexually transmitted infections: 2006 - 2015. Breaking the chain of transmission. Washington: WHO, 2007 